\title{
REVIEW
}

\section{ECLS-associated infections in adults: what we know and what we don't yet know}

\author{
Darryl Abrams ${ }^{1,2}$, Giacomo Grasselli ${ }^{3,4}$, Matthieu Schmidt ${ }^{5,6}$, Thomas Mueller ${ }^{7}$ and Daniel Brodie ${ }^{1,2^{*}}$
}

(c) 2019 Springer-Verlag GmbH Germany, part of Springer Nature

\begin{abstract}
Extracorporeal life support (ECLS) is increasingly used in the management of patients with severe cardiopulmonary disease. Infections are frequently the etiologies underlying the respiratory, and occasionally cardiac, failure that necessitates ECLS. Just as importantly, infections are among the most commonly reported adverse events during ECLS. Infections in this setting may be the sequelae of prolonged critical illness or of underlying immune dysregulation; they may be hospital-acquired infections, and they may or may not be attributable to the presence of ECLS itself, the latter being an aspect that can be difficult to determine. Current registry data and evidence from the literature offer some insights, but also leave open many questions regarding the nature and significance of infections reported both before and during ECLS, including the question of any causal link between ECLS and the development of infections. An ongoing lack of consistency in the identification, diagnosis, management, and prevention of infections during ECLS is limiting our ability to interpret literature data and thus highlighting the need for more rigorous investigation and standardization of definitions. This review aims to characterize the current understanding of infections associated with the use of ECLS, taking into account data from the updated Extracorporeal Life Support Organization Registry, which provides important context for understanding the epidemiology and outcomes of these patients.
\end{abstract}

Keywords: ECLS, ECMO, Infections, Complications, Nosocomial, ELSO registry

\section{Introduction}

The term extracorporeal life support (ECLS) refers to the use of mechanical support devices that provide gas exchange with or without hemodynamic support through an extracorporeal circuit. Blood is drained through a cannula situated in a central vessel (typically a vein), pumped through a membrane, where oxygen is delivered to the blood and carbon dioxide is removed, and then reinfused back through a cannula into a central vessel (vein or artery, depending on the circuit configuration and indication) [1]. Venovenous ECLS is the preferred configuration when there is severe gas exchange impairment,

*Correspondence: hdb5@cumc.columbia.edu

${ }^{1}$ Columbia University College of Physicians and Surgeons, New YorkPresbyterian Hospital, New York, NY, USA

Full author information is available at the end of the article whereas venoarterial ECLS is the appropriate configuration for severe cardiac dysfunction, with or without concomitant respiratory failure $[2,3]$.

Severe forms of acute respiratory distress syndrome (ARDS) are the most common indication for venovenous ECLS, and presumed or proven infectious etiologies typically account for a large proportion of ARDS cases [4-6]. Cardiogenic shock, a condition resulting from a heterogeneous group of diseases, is the most common indication for venoarterial ECLS [1]; again, the underlying etiology may be related to an infectious trigger (e.g., sepsis-associated cardiomyopathy, acute myocarditis, infective endocarditis). A clear understanding of the epidemiology and outcomes associated with the various infectious etiologies of cardiopulmonary failure is essential in order to select the patients most likely to benefit from ECLS. Equally important is the ability to recognize and manage the infectious complications that can arise during ECLS, which may or may not be attributable to the

\section{望 Springer}


device itself, and which may adversely impact outcomes. Administering ECLS involves the placement of intravascular catheters, which introduces a risk of both catheter site infections and catheter-associated blood stream infections [7]. The concomitant use of invasive mechanical ventilation in many of these patients exposes them to the risk of developing ventilator-associated pneumonia (VAP) $[7,8]$. The ECLS population may be particularly susceptible to additional nosocomial infections as a result of concomitant critical illness and associated prolonged hospitalization, which often coincides with the use of other indwelling catheters (e.g., non-ECLS central venous catheters, urinary catheters, etc.) and devices (e.g., continuous renal replacement therapies, intra-aortic balloon pumps, percutaneous ventricular assist devices) [9]. Furthermore, underlying comorbidities, critical illness, or perhaps even the extracorporeal circuit itself, may cause immune dysregulation, potentially leading to a higher rate of infectious complications. The management of infections during ECLS is made even more challenging by alterations in the pharmacokinetics and pharmacodynamics of antimicrobial agents in the presence of both critical illness and extracorporeal circuitry [10, 11]. This narrative review aims to characterize the current understanding of infections associated with ECLS, discuss the challenges in identifying and treating these infections, and highlight areas where more data are needed to better understand the susceptibility to and impact of infections in patients managed with ECLS.

\section{Infections prior to the initiation of ECLS}

Both bacterial and viral pneumonia, when leading to severe acute respiratory failure, are common indications for venovenous ECLS [4-6, 12]. With regard to specific infectious organisms, one of the most widely reported indications for ECLS in ARDS is influenza A (H1N1), partly as a result of the rapid increase in the use of ECLS during the 2009 H1N1 pandemic. Data from several large observational studies suggest, overall, that there is a probable benefit to be derived from the use of ECLS in H1N1-associated severe ARDS refractory to conventional therapy [13-15]. Whereas no specific infectious etiology has been identified as a contraindication to the use of ECLS, co-infections in the context of influenzaassociated ARDS, including infections with $S$. aureus, multidrug-resistant Gram-negative bacteria and Aspergillus, may correlate with lower survival compared with influenza alone [16-19]. Likewise, concomitant nonpulmonary infections at the time of initiation of ECLS for ARDS may predict worse outcomes and this factor has been incorporated into a validated prognostic scoring system [20].

\section{Take-home message}

Infections commonly precede and are frequently identified during ECLS. The current paucity of knowledge about the epidemiology and outcomes of ECLS-associated infections highlights the need for standardized definitions, consistent detection strategies, and more data, which might allow meaningful conclusions to be drawn regarding the clinical significance of these infections, and inform best practices for their prevention and management.

While infectious etiologies may account for a relatively small proportion of the indications for venoarterial ECLS in cardiac failure, certain subpopulations warrant consideration. Both acute myocarditis, which is often attributable to infection, and sepsis-associated cardiomyopathy are indications that may be associated with favorable survival rates [21-23].

The Extracorporeal Life Support Organization (ELSO) registry is the largest international repository of epidemiological data on ECLS. The results of an ELSO registry search for all infectious organisms identified on culture prior to the initiation of ECLS between January 1, 2012 (correlating with a reformatting of data entry within the registry for more extensive detail on infections) and July 31, 2019 are summarized in Figs. 1 and 3a, and in the Online Resource, with the most common pathogens listed by frequency. These data should be interpreted with caution, as culture positivity may not represent true infection, and the presence of these organisms prior to the initiation of ECLS may or may not have contributed to the cardiopulmonary failure that prompted the use of ECLS. Furthermore, when interpreting infections identified during ECLS, it is important to take into account any pre-ECLS infections; indeed, without a thorough understanding of what was present prior to ECLS, any pre-existing infections may incorrectly be characterized as de novo ones. With regard to the frequency of positive cultures, represented as a percentage of total ECLS runs, it is important to remember that individual patients may be represented by more than one culture. Additionally, registry data is self-reported by ELSO member centers, and may therefore be influenced by variability in data collection and in interpretation of data definitions; furthermore, these data, even if accurate, may not be representative of ECLS practices at non-ELSO-affiliated sites. Albeit subject to these important limitations and caveats, the registry data search revealed 5492 positive cultures prior to the initiation of 17,374 distinct ECLS runs for respiratory failure (31.6\%). Positive cultures prior to ECLS appeared to be much more common in the case of ECLS administered for respiratory failure than for cardiac indications (8.8\% of cardiac failure runs; $7 \%$ of runs for extracorporeal cardiopulmonary resuscitation 


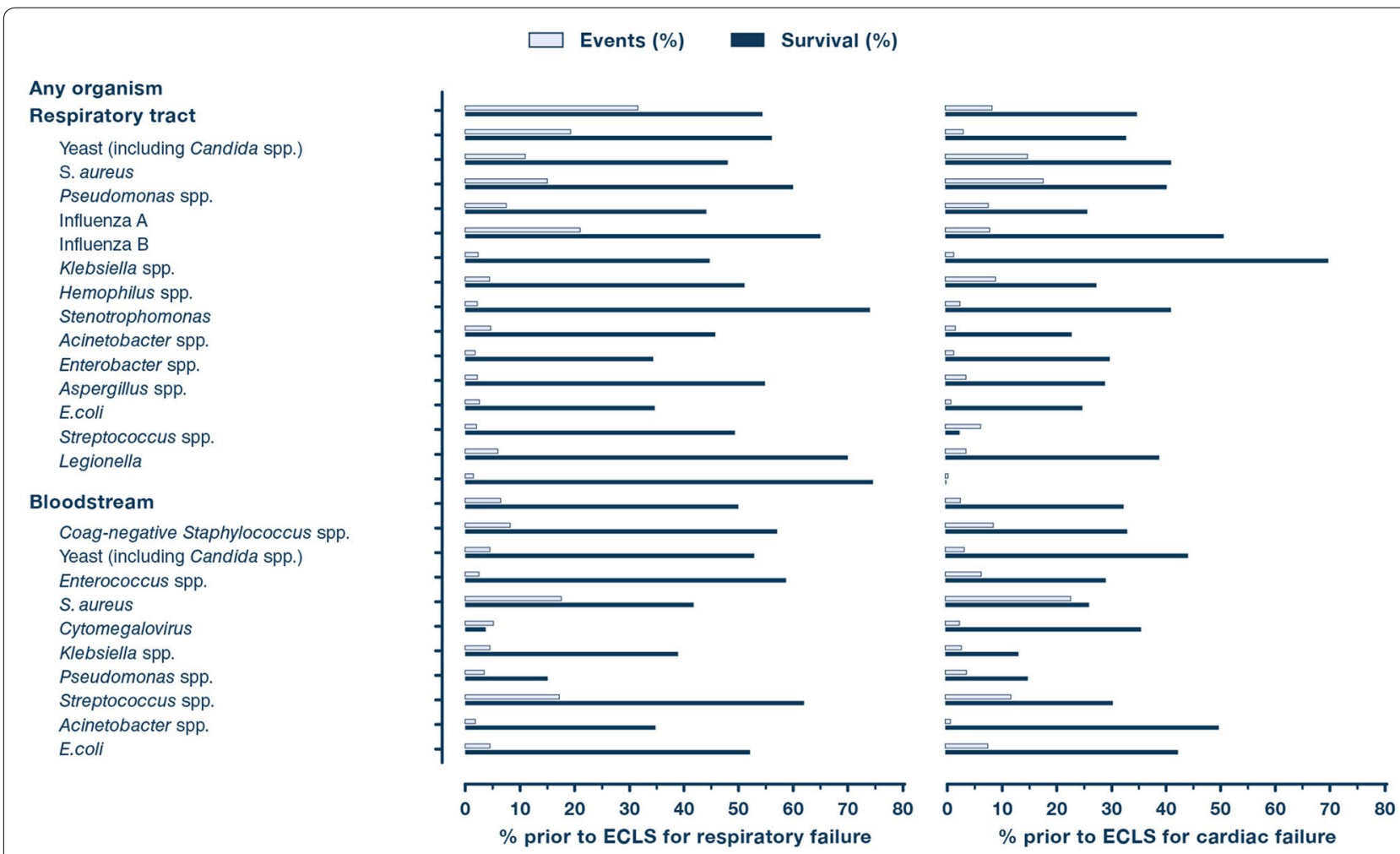

Fig. 1 Most common organisms identified on culture prior to initiation of ECLS for respiratory and cardiac failure. Based on a total of 17,374 ECLS runs for respiratory failure and a total of 18,514 total ECLS runs for cardiac failure from January 2012 through July 2019. Event rate represents the number of positive cultures for a given organism divided by the total number of ECLS runs. ECLS extracorporeal life support

(ECPR)), with the respiratory tract being the most frequent site throughout.

Regardless of ECLS indication, $S$. aureus and yeast were consistently among the most common respiratory tract pathogens reported. Notably, Influenza A was the most commonly identified organism in the respiratory tract in patients receiving ECLS for respiratory failure, accounting for $12.7 \%$ of all organisms identified. Staphylococcus and Streptococcus were the most common isolates in the bloodstream across all indications. Yeast, E. coli, and Enterococcus were common pathogens in the urine.

\section{Infections during ECLS}

Reported rates of hospital-acquired infections during ECLS have varied substantially across case series and registries; these data have inherent limitations due to variations in definitions, reporting methods, and surveillance practices. Patients receiving ECLS may be at risk of developing typical ICU-related nosocomial infections (e.g., VAP, bloodstream infections, urinary tract infections), in addition to ECLS-specific infections, such as localized infections at peripheral cannulation insertion sites or mediastinitis in the setting of central cannulation $[7,24]$. Bizzarro et al. [25] reported a $21 \%$ prevalence rate of nosocomial infections among adults recorded in the ELSO registry from 1998-2008, whereas previous case series estimated nosocomial infection rates of between 9 and 65\% during ECLS [7, 26-29].

Figures 2 and $3 \mathrm{~b}$ and the Online Resource summarize the ELSO registry data documenting positive cultures during ECLS administered for respiratory failure, cardiac failure, and cardiac arrest. ECLS for respiratory failure showed the highest rate of positive cultures during ECLS (64.9\%), whereas ECPR showed the lowest rate (22\%). The distribution of pathogens during ECLS was found to be similar to the pre-ECLS pattern. Yeast and $S$. aureus were the most common organisms in the respiratory tract during ECLS across all three groups, whereas Staphylococcus, yeast, and Enterococcus were among the organisms isolated most frequently from blood cultures. The survival rates of individuals with positive cultures during ECLS administered for respiratory failure or cardiac failure were lower than the overall survival rates of all ECLS recipients over a similar timeframe $(54.3 \%$ vs $61.1 \%$ and $38.0 \%$ vs $44.2 \%$ ) [12], whereas survival among culture-positive ECPR patients was the lowest of the three groups but comparable to that observed in the general ECPR population (30.2\% vs $29.9 \%)$ [12]. Although 

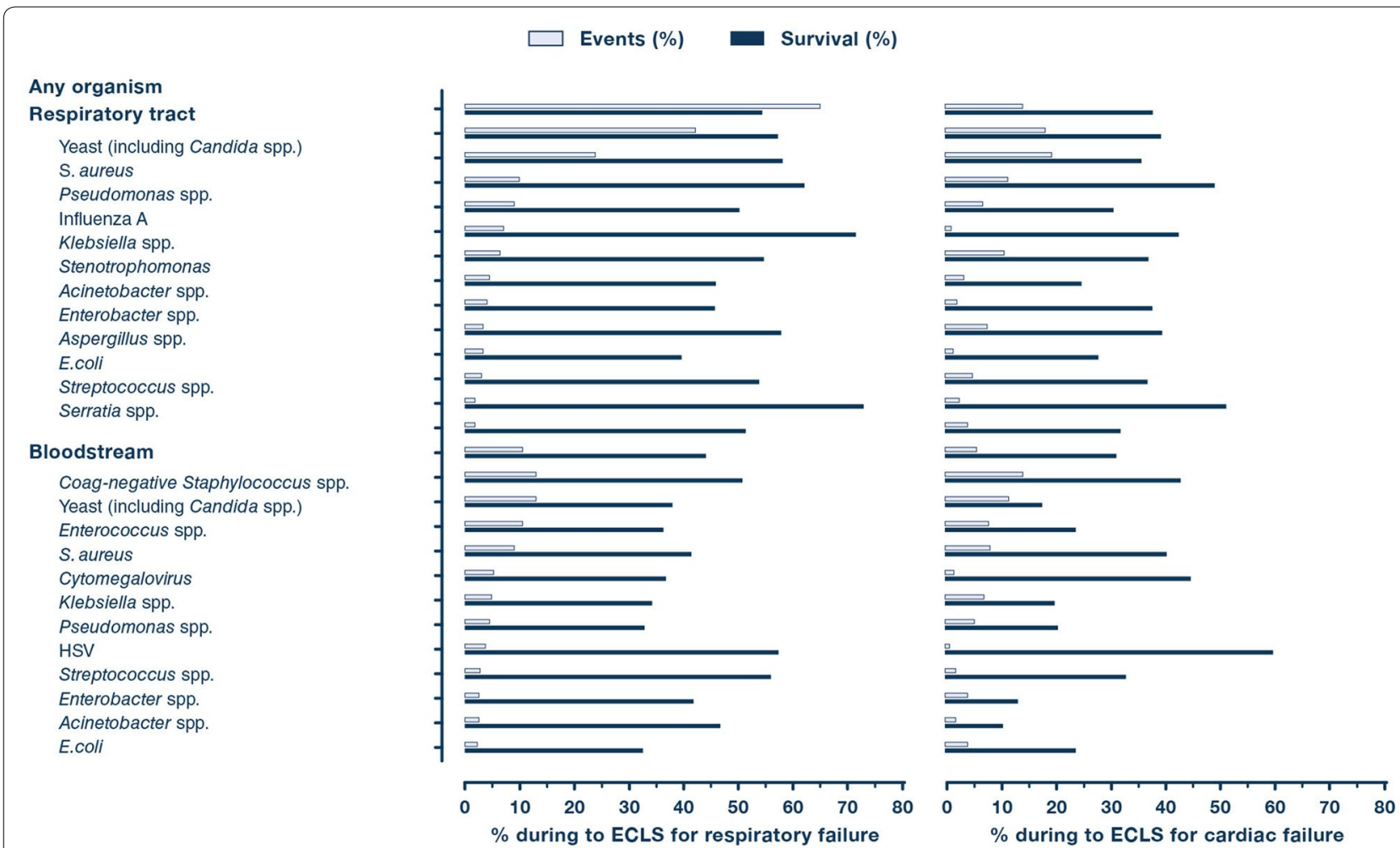

Fig. 2 Most common organisms identified on culture during ECLS for respiratory and cardiac failure. Based on a total of 17,374 total ECLS runs for respiratory failure and a total of 18,514 total ECLS runs for cardiac failure from January 2012 through July 2019. Event rate represents the number of positive cultures for a given organism divided by the total number of ECLS runs. ECLS extracorporeal life support

these data seem to suggest a correlation between acquisition of nosocomial infections and increased mortality, in the absence of further information regarding underlying diagnoses, comorbid conditions, and other factors that undoubtedly confound this association, only tentative conclusions can be drawn.

It is important to reiterate that these data refer only to organisms identified on culture and are not necessarily indicative of active infections. Some of these organisms may represent colonizations without pathological significance (e.g., the detection of Candida in the respiratory tract). Similarly, cultures obtained during ECLS may show the persistence of an organism already present before ECLS initiation (as in the case, for example, of a patient with $S$. aureus in the bloodstream before ECLS initiation who remains bacteremic during ECLS) rather than necessarily being indicative of newly identified organisms or a new infection. That said, the ELSO registry continues to provide the most comprehensive and detailed body of data available on the prevalence of infections during ECLS.

Beyond registry data, the value of primary investigational data from individual sites is often limited by methodology and generalizability issues. Having said that, a recent single-center observational study of 92 patients receiving ECLS (87\% venovenous ECLS), in whom infections were systematically and prospectively identified through application of well-established clinical practice guidelines [30,31], reported high rates of nosocomial infections (55\%), with VAP and multidrugresistant organisms found to be common [24]. Those who acquired nosocomial infections had higher overall mortality, longer durations of mechanical ventilation and ECLS, and spent longer in the ICU.

\section{Risk factors for the development of infections during ECLS}

The etiology of nosocomial infections during ECLS has not been systematically compared with (although it likely parallels) that of infections acquired in the course of critical illness in the absence of ECLS; however it remains to be established whether certain characteristics unique to patients receiving ECLS confer an increased risk of infection. This can only be speculated upon from the existing data (Table 1). It has been suggested, initially in patients undergoing cardiopulmonary bypass, that extracorporeal circuitry affects the immune system through multiple mechanisms (e.g., induction of endothelial dysfunction, 

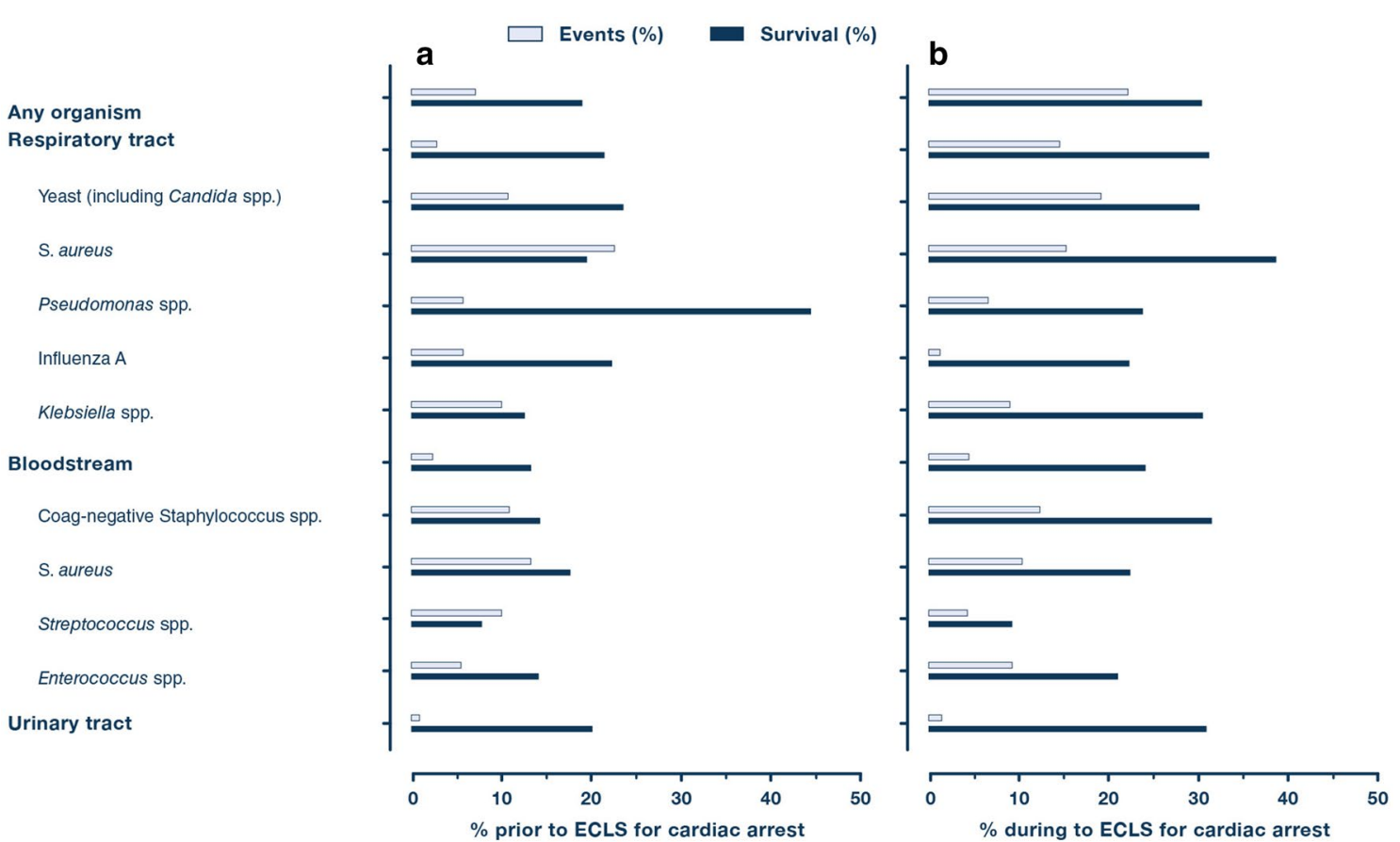

Fig. 3 Most common organisms identified on culture prior to initiation of ECLS (a) and during ECLS (b) for cardiac arrest. Based on a total of 5979 ECLS runs for cardiac arrest from January 2012 through July 2019. Event rate represents the number of positive cultures for a given organism divided by the total number of ECLS runs. ECLS extracorporeal life support

\section{Table 1 Potential risk factors for infections during ECLS}

Immunocompromised status (including potential immune dysregulation from the presence of ECLS itself)

\section{Prolonged duration of ECLS ${ }^{a}$}

Older age $^{\mathrm{a}}$

Higher pre-ECLS severity of illness ${ }^{a}$

Underlying autoimmune disorder

Central cannulation (vs peripheral cannulation)

Surgical cannulation approach (vs percutaneous approach)

ECLS extracorporeal life support

a There are no specific cutoffs, per se, but the longer the duration of ECLS, the older the age, and the higher the pre-ECLS severity of illness, the higher the presumed risk

activation of the contact system, coagulation cascade, neutrophils and platelets with consequent release of pro-inflammatory mediators) [32], and that the resulting immune system impairment might explain why ECLS may increase susceptibility to infection [33]. In contrast to this supposed hyperinflammatory response to ECLS, there exist equally compelling data suggesting that ECLS promotes an anti-inflammatory state through improvements in end-organ perfusion and gas exchange in patients with severe cardiopulmonary failure and reductions in pro-inflammatory injury (e.g., ventilator-induced lung injury) $[34,35]$. A prospective observational study of 262 adult patients with severe ARDS reported a rapid decline in IL- 6 and IL-8 levels within $24 \mathrm{~h}$ of the start of venovenous ECLS. Higher cytokine levels were associated with extra-pulmonary causes of ARDS, more aggressive ventilation before ECLS, and mortality [36]. The overall balance between pro- and anti-inflammatory effects (i.e., whether end-organ protective strategies outweigh the injurious effects of ECLS) likely determine the impact on the immune response and the consequent risk of infection. Whether these alterations pose an increased risk of infection after decannulation from ECLS is an area that warrants further investigation [37].

Whether or not the presence of ECLS leads to additional immune system impairment, immunocompromised patients appear to have worse outcomes than immunocompetent patients. In a multicenter observational study of 203 immunocompromised patients receiving ECLS for severe ARDS, nosocomial infections were common, with VAP diagnosed in $50 \%$ of patients and cannula-associated infections (defined as local signs of infection at a cannula site with positive culture from subcutaneous needle aspirate) found in $10 \%$ of patients [6]. With 6-month survival rates standing at only $30 \%$, particular consideration should be given to preventive practices and close surveillance of infections during ECLS in 
this vulnerable patient population. Of note, the acquisition of nosocomial infections was not an independent predictor of mortality in multivariable analysis.

While ECLS duration has frequently been associated with the development of nosocomial infections [7, 2629], no existing analyses are able to determine whether longer duration of support is a risk factor for or a consequence of acquired infections. Prolonged use of ECLS will increase the opportunity to acquire infections, and infections are likely to contribute to, and increase the duration of, critical illness. Any relationship found between nosocomial infections and duration of ECLS should be interpreted with particular caution, as patients who acquire and die from infections early in their hospitalization would obviously have had shorter ECLS durations.

Older age, higher pre-ECLS severity of illness, underlying autoimmune disorders, circuit configurations, and performance of procedures during ECLS have all been implicated as risk factors for infections during ECLS [24, $27-29,38,39]$. However, given the predominantly singlecenter, retrospective nature of the cited studies, the possibility of determining the true incremental risk, if any, deriving from these factors is limited and warrants more systematic investigation. Of note, Grasselli et al. identified younger, not older, age as being independently associated with higher risk of nosocomial infections, with VAP and multidrug-resistant organisms associated with higher mortality [24].

Whether the site and number of ECLS cannulae is associated with increased infection risk has yet to be thoroughly investigated. There is weak evidence suggesting an increased risk of infection with central cannulation and a decreased risk with single-site, dual-lumen cannulae [7, 38]. In a recent single-center propensity score-matched analysis of 814 patients undergoing venoarterial ECLS, cannula site infections were significantly more common with a surgical approach than a percutaneous approach $(27.8 \%$ vs $16.5 \%, p<0.001)$ [40]. Historical data suggest an increased incidence of bloodstream infections in ECLS patients compared with non-ECLS critically ill patients [41], although this has not been corroborated in the era of modern ECLS technology and practices. Cannulae and membranes are both potential surfaces for microbial colonization, as demonstrated by several studies [42-44]. Whether these surfaces represent primary or secondary sites of infection is not currently known.

\section{Challenges in the detection, treatment, and prevention of infections during ECLS}

Efforts to detect infections during ECLS come up against particular difficulties that could impede their prompt recognition and treatment, as well as the assessment of response to antimicrobial therapy. Passive or active cooling or heating of the blood as it passes through the membrane may mask fever or hypothermia [11]. The presence of infiltrates on chest radiographs, used to help identify VAP [31], may be difficult to interpret when extreme lung-protective ventilatory strategies are employed, as these result in extensive airspace opacification in the absence of a new infection [13], or in cases of severe cardiogenic pulmonary edema. The potential for ECLS to affect serological tests used in the detection of infections, much in the way cellulose membranes may elevate $1,3-\beta$-D-glucan levels [45], is an important consideration that warrants further investigation. The difficulty in detecting infection by conventional means may influence surveillance practices, as highlighted by a high reported rate of daily blood cultures performed as routine surveillance in a survey of ELSO-affiliated ECLS centers [46].

The pharmacokinetics and pharmacodynamics of antimicrobial agents used to treat infections during ECLS may be affected by several factors associated with both critical illness and the ECLS circuit itself, including clearance abnormalities in the setting of organ failures, potential for sequestration within the circuit, and increases in the volume of distribution (Vd) [10, 11, 47]. The lipophilicity and protein binding characteristics of a given drug play an important role in its interaction with the circuit [11]. Several antibiotics have been studied in the context of ECLS, and shown varying susceptibility to subtherapeutic levels due to sequestration or increased Vd; the results have been summarized elsewhere $[10,11]$. When available, therapeutic drug monitoring should be performed to ensure adequate dosing of medications during ECLS.

Despite a lack of data demonstrating benefits, and in spite of ELSO Infectious Disease Task Force recommendations against the practice, antibiotic prophylaxis is commonly used at ECLS centers, according to surveys of ELSO member sites [46, 48, 49]. Whether or not antibiotic, or antifungal, prophylaxis is warranted or affects the incidence of nosocomial infections may become moot if most patients are already receiving antimicrobial agents at the time of initiation of ECLS. For example, $97 \%$ of patients in the previously mentioned cohort of immunocompromised patients were receiving antibiotics before ECLS, despite in many cases not having an infectious etiology for their respiratory failure [6]. Other infection prevention practices, including cannula maintenance strategies, vary across centers $[46,50]$. Many of the recommendations put forth by ELSO, such as the use of sterile techniques, adherence to VAP prevention guidelines, and avoidance or removal of unnecessary invasive devices, mirror general infection prevention practices followed in critically ill patients (Fig. 4) [26, 49]. One 


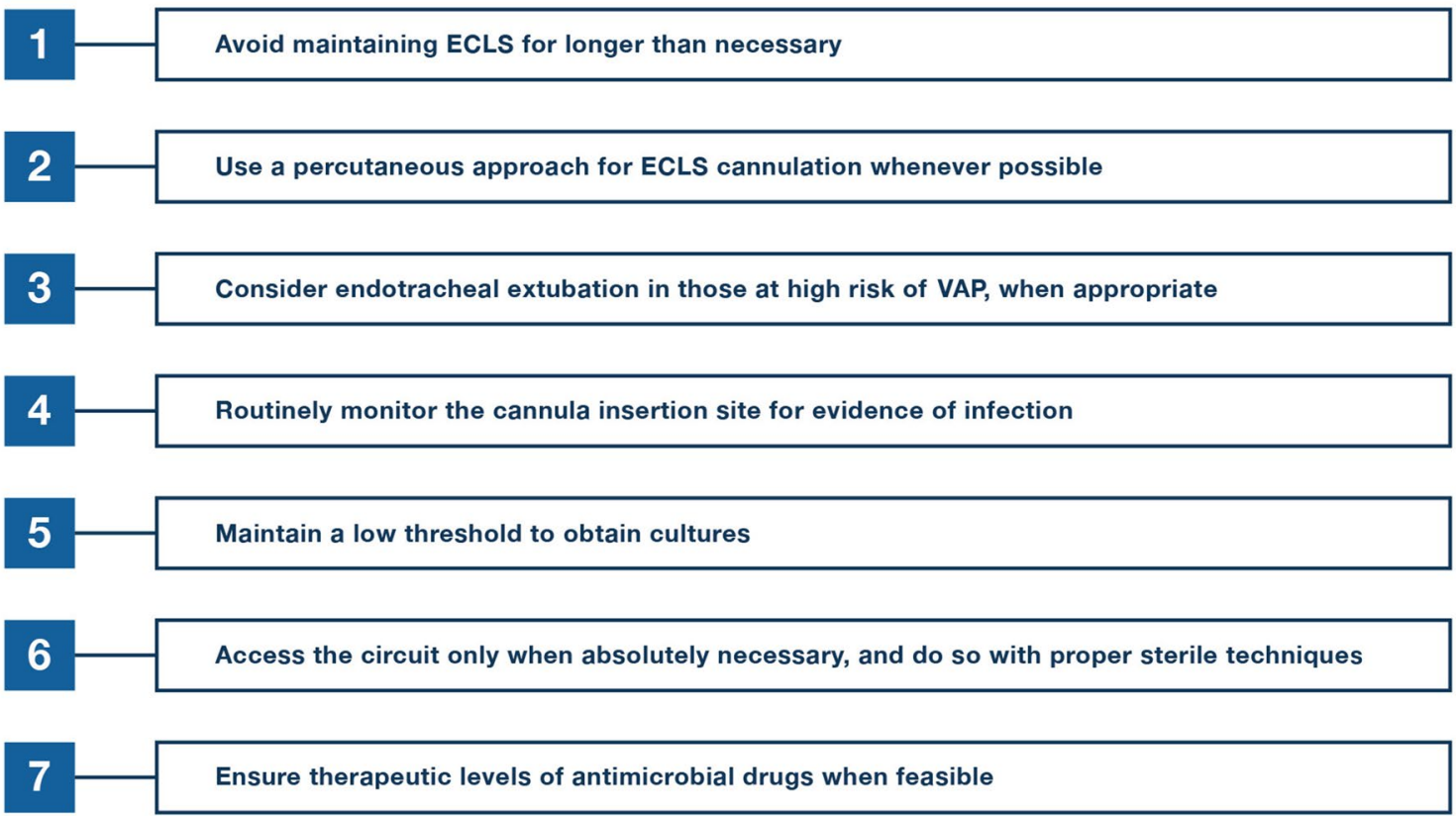

Fig. 4 Potential strategies to reduce infections during ECLS. ECLS extracorporeal life support, VAP ventilator-associated pneumonia

emerging VAP prevention strategy that may be used in selected ECLS patients is endotracheal extubation with removal of invasive mechanical ventilation. Patients with primarily cardiac failure, who have relatively preserved gas exchange, patients with predominantly hypercapnic respiratory failure, or those in whom early mobilization is of greater importance to their ultimate recovery, such as patients receiving ECLS as a bridge to transplantation, may be particularly suitable for such a strategy [51]. The roles of other infection prevention strategies that have been explored in general critical care populations, such as selective digestive decontamination, remain uncertain in ECLS patients.

\section{Future research directions}

The paucity of high-quality data on ECLS-associated infections highlights the need for a more systematic evaluation of their incidence, outcomes and risk factors, and of appropriate prevention approaches and treatment strategies (Fig. 5). Without consistent definitions of ECLS-associated infections (including infections attributed to the device itself) and standardized reporting of these events, it remains impossible to quantify the true incidence of infections both before and after the initiation of ECLS, including infections acquired after ECLS removal. A better understanding of infection susceptibility during ECLS would help to clarify whether antimicrobial prophylaxis or routine surveillance cultures might be warranted in certain patient populations, or perhaps whether ECLS should be avoided altogether in the presence of infections with universally poor outcomes.

A systematic review has previously characterized inconsistencies existing in the definition and reporting of infectious complications in venoarterial ECLS [52], highlighting the need for methodologically rigorous development of consensus opinions regarding infectious complications and associated outcomes in order to obtain accurate and clinically meaningful epidemiological data [53]. The ELSO registry is currently undergoing a redesign that may allow for more granularity regarding infectious indications and complications [54].

Ideally, in order to be generalizable to the broader ECLS community, studies evaluating the epidemiology of ECLS-associated infections should be performed across diverse patient populations with varying rates of organism prevalence and antimicrobial resistance, using standardized definitions of infections, and systematically measuring important confounders that may contribute to infections and outcomes. Along such lines, a multicenter, prospective observational study evaluating the prevalence of nosocomial infections and cannula management practices in ECLS across Australia and New Zealand has recently been proposed [55]. Research networks, such as the International ECMO Network (ECMONet; www. internationalecmonetwork.org), provide a forum for performing this type of research in ECLS across centers and regions. In the meantime, we recommend that all centers 
- Is there a role for antibiotic prophylaxis during cannulation and/or decannulation?

- Is there a role for obtaining routine surveillance cultures?

- Should chlorhexidine-impregnated cannula dressings be used?

- Should there be a standardized ECLS infection prevention bundle?

Prevention

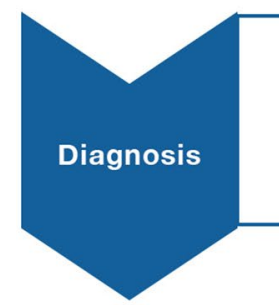

- How should cannula-associated infections be defined?

- Are subcutaneous needle aspirates or skin swabs needed for diagnosis of cannula-associated infections?

- Should cannula tip cultures routinely be obtained upon decannulation?

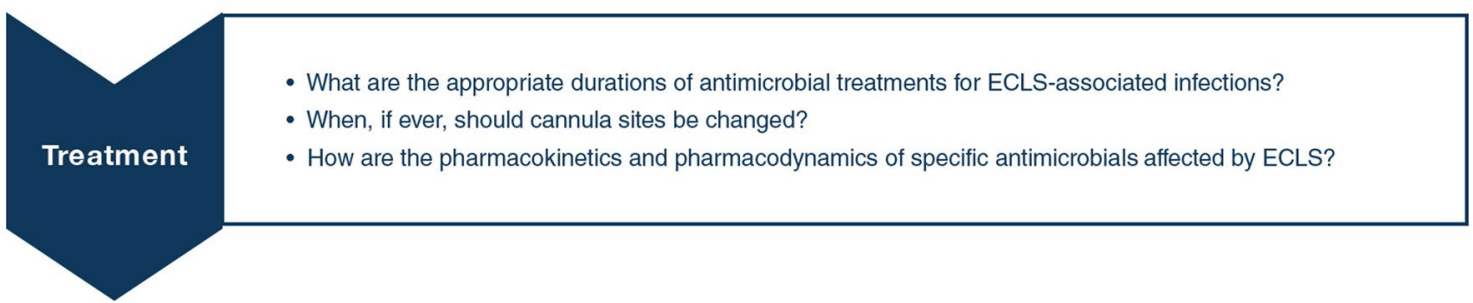

Fig. 5 Research agenda and ongoing uncertainties regarding ECLS-associated infections. ECLS extracorporeal life support

performing ECLS enter their data into the ELSO or other equivalent registry.

In addition to epidemiological data, more information is needed regarding antimicrobial pharmacokinetics and pharmacodynamics during ECLS in order to optimize treatment of peri-ECLS infections. The ongoing Analgesia, Sedation, and Antibiotic Pharmacokinetics during Extracorporeal Membrane Oxygenation (ASAP ECMO) study hopes to provide additional insight into the impact of ECLS on the efficacy of antimicrobials [56].

\section{Conclusions}

Infections both commonly precede and are frequently identified during ECLS. This review of current knowledge regarding ECLS-associated infections highlights the need for standardized definitions, consistent detection strategies, and more comprehensive descriptions of patient characteristics and outcomes so that meaningful conclusions regarding the clinical significance of these infections can be drawn and inform best practices for their prevention and management.

\section{Electronic supplementary material}

The online version of this article (https://doi.org/10.1007/s00134-019-05847-z) contains supplementary material, which is available to authorized users.

\section{Author details}

${ }^{1}$ Columbia University College of Physicians and Surgeons, New York-Presbyterian Hospital, New York, NY, USA. ${ }^{2}$ Center for Acute Respiratory Failure, Columbia University Medical Center, New York, NY, USA. ${ }^{3}$ Department of Anesthesia, Critical Care and Emergency, Fondazione IRCCS Ca' Granda Ospedale Maggiore Policlinico, Milan, Italy. ${ }^{4}$ Department of Pathophysiology and Transplantation, University of Milan, Milan, Italy. ${ }^{5}$ Sorbonne Université, INSERM, UMRS_1166ICAN, Paris, France. ${ }^{6}$ Service de médecine Intensive-réanimation, Institut de Cardiologie, APHP Hôpital Pitié-Salpêtrière, 75013 Paris, France. ${ }^{7}$ Department of Internal Medicine II, University Hospital of Regensburg, Regensburg, Germany.

\section{Acknowledgements}

We would like to thank Peter Rycus, Peta Alexander, and the Extracorporeal Life Support Organization for their assistance in providing data from the ELSO Registry.

\section{Compliance with ethical standards}

\section{Conflicts of interest}

MS has received lectures fees from Getinge, Xenios and Dräger, outside the submitted work. DB reports fees to his university from ALung Technologies, personal fees from Baxter and anticipated fees from BREETHE, an unpaid association with Hemovent, outside the submitted work. GG received payment for lectures from Draeger Medical, Getinge, Fisher and Paykel, Pfizer, and received travel/accommodations/congress registration support from Getinge and Biotest, outside the submitted work.

\section{Publisher's Note}

Springer Nature remains neutral with regard to jurisdictional claims in published maps and institutional affiliations.

Received: 5 September 2019 Accepted: 30 October 2019 Published online: 25 November 2019 


\section{References}

1. Abrams D, Combes A, Brodie D (2014) Extracorporeal membrane oxygenation in cardiopulmonary disease in adults. J Am Coll Cardiol 63:2769-2778

2. Brodie D, Slutsky AS, Combes A (2019) Extracorporeal life support for adults with respiratory failure and related indications: a review. JAMA 322:557-568

3. Abrams D, Garan AR, Abdelbary A, Bacchetta M, Bartlett RH, Beck J, Belohlavek J, Chen YS, Fan E, Ferguson ND, Fowles JA, Fraser J, Gong M, Hassan IF, Hodgson C, Hou X, Hryniewicz K, Ichiba S, Jakobleff WA, Lorusso R, MacLaren G, McGuinness S, Mueller T, Park PK, Peek G, Pellegrino V, Price S, Rosenzweig EB, Sakamoto T, Salazar L, Schmidt M, Slutsky AS, Spaulding C, Takayama H, Takeda K, Vuylsteke A, Combes A, Brodie D, International EN, The Extracorporeal Life Support O (2018) Position paper for the organization of ECMO programs for cardiac failure in adults. Intensive Care Med 44:717-729

4. Thiagarajan RR, Barbaro RP, Rycus PT, McMullan DM, Conrad SA, Fortenberry JD, Paden ML, ELSO member centers (2017) Extracorporeal life support organization registry international report 2016. ASAIO J 63:60-67

5. Schmidt M, Pham T, Arcadipane A, Agerstrand C, Ohshimo S, Pellegrino V, Vuylsteke A, Guervilly C, McGuinness S, Pierard S, Breeding J, Stewart C, Ching SSW, Camuso JM, Stephens RS, King B, Herr D, Schultz MJ, Neuville M, Zogheib E, Mira JP, Roze H, Pierrot M, Tobin A, Hodgson C, Chevret S, Brodie D, Combes A (2019) Mechanical ventilation management during extracorporeal membrane oxygenation for acute respiratory distress syndrome. An international multicenter prospective cohort. Am J Respir Crit Care Med 200:1002-1012

6. Schmidt M, Schellongowski P, Patroniti N, Taccone FS, Reis Miranda D, Reuter J, Prodanovic H, Pierrot M, Dorget A, Park S, Balik M, Demoule A, Crippa IA, Mercat A, Wohlfarth P, Sonneville R, Combes A, International Ecmo Network tRRN, the ISG (2018) Six-month outcome of immunocompromised patients with severe acute respiratory distress syndrome rescued by ECMO. An international multicenter retrospective study. Am J Respir Crit Care Med 197:1297-1307

7. Schmidt M, Brechot N, Hariri S, Guiguet M, Luyt CE, Makri R, Leprince P, Trouillet JL, Pavie A, Chastre J, Combes A (2012) Nosocomial infections in adult cardiogenic shock patients supported by venoarterial extracorporeal membrane oxygenation. Clin Infect Dis 55:1633-1641

8. Bougle A, Bombled C, Margetis D, Lebreton G, Vidal C, Coroir M, Hajage D, Amour J (2018) Ventilator-associated pneumonia in patients assisted by veno-arterial extracorporeal membrane oxygenation support: epidemiology and risk factors of treatment failure. PLoS One 13:e0194976

9. Husain-Syed F, Ricci Z, Brodie D, Vincent JL, Ranieri VM, Slutsky AS, Taccone FS, Gattinoni L, Ronco C (2018) Extracorporeal organ support (ECOS) in critical illness and acute kidney injury: from native to artificial organ crosstalk. Intensive Care Med 44:1447-1459

10. Dzierba AL, Abrams D, Brodie D (2017) Medicating patients during extracorporeal membrane oxygenation: the evidence is building. Crit Care 21:66

11. Dzierba AL, Abrams D, Muir J, Brodie D (2019) Ventilatory and pharmacotherapeutic strategies for management of adult patients on extracorporeal life support. Pharmacotherapy 39:355-368

12. International Summary of the ECMO Registry of the Extracorporeal Life Support Organization (ELSO) (2019) Ann Arbor, Michigan. https://www. elso.org/Registry/Statistics/InternationalSummary.aspx. Accessed 20 Aug 2019

13. Davies A, Jones D, Bailey M, Beca J, Bellomo R, Blackwell N, Forrest $P$, Gattas D, Granger E, Herkes R, Jackson A, McGuinness S, Nair P, Pellegrino V, Pettila V, Plunkett B, Pye R, Torzillo P, Webb S, Wilson M, Ziegenfuss M (2009) Extracorporeal membrane oxygenation for 2009 influenza A(H1N1) acute respiratory distress syndrome. JAMA 302:1888-1895

14. Pham T, Combes A, Roze H, Chevret S, Mercat A, Roch A, Mourvillier B, Ara-Somohano C, Bastien O, Zogheib E, Clavel M, Constan A, Marie Richard JC, Brun-Buisson C, Brochard L (2013) Extracorporeal membrane oxygenation for pandemic influenza $A(\mathrm{H} 1 \mathrm{~N} 1)$-induced acute respiratory distress syndrome: a cohort study and propensity-matched analysis. Am J Respir Crit Care Med 187:276-285

15. Noah MA, Peek GJ, Finney SJ, Griffiths MJ, Harrison DA, Grieve R, Sadique MZ, Sekhon JS, McAuley DF, Firmin RK, Harvey C, Cordingley JJ, Price S, Vuylsteke A, Jenkins DP, Noble DW, Bloomfield R, Walsh TS, Perkins GD, Menon D, Taylor BL, Rowan KM (2011) Referral to an extracorporeal membrane oxygenation center and mortality among patients with severe 2009 influenza A(H1N1). JAMA 306:1659-1668

16. Rozencwajg S, Brechot N, Schmidt M, Hekimian G, Lebreton G, Besset S, Franchineau G, Nieszkowska A, Leprince P, Combes A, Luyt CE (2018) Coinfection with influenza-associated acute respiratory distress syndrome requiring extracorporeal membrane oxygenation. Int J Antimicrob Agents 51:427-433

17. De Rosa FG, Corcione S, Pagani N, Stella ML, Urbino R, Di Perri G, Ranieri VM (2013) High rate of respiratory MDR gram-negative bacteria in H1N1ARDS treated with ECMO. Intensive Care Med 39:1880-1881

18. van de Veerdonk FL, Kolwijck E, Lestrade PP, Hodiamont CJ, Rijnders BJ, van Paassen J, Haas PJ, Oliveira Dos Santos C, Kampinga GA, Bergmans DC, van Dijk K, de Haan AF, van Dissel J, van der Hoeven HG, Verweij PE, Dutch Mycoses Study G (2017) Influenza-Associated Aspergillosis in Critically III Patients. Am J Respir Crit Care Med 196:524-527

19. Jacquot A, Luyt CE, Kimmoun A, Levy B, Baux E, Fluvalentine Study g (2019) Epidemiology of post-influenza bacterial pneumonia due to Panton-Valentine leucocidin positive Staphylococcus aureus in intensive care units: a retrospective nationwide study. Intensive Care Med 45:1312-1314

20. Schmidt M, Bailey M, Sheldrake J, Hodgson C, Aubron C, Rycus PT, Scheinkestel C, Cooper DJ, Brodie D, Pellegrino V, Combes A, Pilcher D (2014) Predicting survival after extracorporeal membrane oxygenation for severe acute respiratory failure. The respiratory extracorporeal membrane oxygenation survival prediction (RESP) score. Am J Respir Crit Care Med 189:1374-1382

21. Schmidt M, Burrell A, Roberts L, Bailey M, Sheldrake J, Rycus PT, Hodgson C, Scheinkestel C, Cooper DJ, Thiagarajan RR, Brodie D, Pellegrino V, Pilcher D (2015) Predicting survival after ECMO for refractory cardiogenic shock: the survival after veno-arterial-ECMO (SAVE)-score. Eur Heart J 36:2246-2256

22. Combes A, Leprince $P$, Luyt CE, Bonnet N, Trouillet JL, Leger P, Pavie A, Chastre J (2008) Outcomes and long-term quality-of-life of patients supported by extracorporeal membrane oxygenation for refractory cardiogenic shock. Crit Care Med 36:1404-1411

23. Brechot $N$, Luyt CE, Schmidt M, Leprince P, Trouillet JL, Leger P, Pavie A, Chastre J, Combes A (2013) Venoarterial extracorporeal membrane oxygenation support for refractory cardiovascular dysfunction during severe bacterial septic shock. Crit Care Med 41:1616-1626

24. Grasselli G, Scaravilli V, Di Bella S, Biffi S, Bombino M, Patroniti N, Bisi L, Peri AM, Pesenti A, Gori A, Alagna L (2017) Nosocomial infections during extracorporeal membrane oxygenation: incidence, etiology, and impact on patients' outcome. Crit Care Med 45:1726-1733

25. Bizzarro MJ, Conrad SA, Kaufman DA, Rycus P, Extracorporeal Life Support Organization Task Force on Infections EMO (2011) Infections acquired during extracorporeal membrane oxygenation in neonates, children, and adults. Pediatr Crit Care Med 12:277-281

26. Biffi S, Di Bella S, Scaravilli V, Peri AM, Grasselli G, Alagna L, Pesenti A, Gori A (2017) Infections during extracorporeal membrane oxygenation: epidemiology, risk factors, pathogenesis and prevention. Int J Antimicrob Agents 50:9-16

27. Sun HY, Ko WJ, Tsai PR, Sun CC, Chang YY, Lee CW, Chen YC (2010) Infections occurring during extracorporeal membrane oxygenation use in adult patients. J Thorac Cardiovasc Surg 140(1125-1132):e1122

28. Pieri M, Agracheva N, Fumagalli L, Greco T, De Bonis M, Calabrese MC, Rossodivita A, Zangrillo A, Pappalardo F (2013) Infections occurring in adult patients receiving mechanical circulatory support: the two-year experience of an Italian National Referral Tertiary Care Center. Medicina Intensiva 37:468-475

29. Aubron C, Cheng AC, Pilcher D, Leong T, Magrin G, Cooper DJ, Scheinkestel C, Pellegrino V (2013) Infections acquired by adults who receive extracorporeal membrane oxygenation: risk factors and outcome. Infect Control Hosp Epidemiol 34:24-30

30. Mermel LA, Allon M, Bouza E, Craven DE, Flynn P, O'Grady NP, Raad II, Rijnders BJ, Sherertz RJ, Warren DK (2009) Clinical practice guidelines for the diagnosis and management of intravascular catheter-related infection: 2009 Update by the Infectious Diseases Society of America. Clin Infect Dis 49:1-45

31. Kalil AC, Metersky ML, Klompas M, Muscedere J, Sweeney DA, Palmer LB, Napolitano LM, O'Grady NP, Bartlett JG, Carratala J, El Solh AA, Ewig S, Fey PD, File TM Jr, Restrepo MI, Roberts JA, Waterer GW, Cruse P, Knight SL, Brozek JL (2016) Management of adults with hospital-acquired and 
ventilator-associated pneumonia: 2016 clinical practice guidelines by the Infectious Diseases Society of America and the American Thoracic Society. Clin Infect Dis 63:e61-e111

32. Millar JE, Fanning JP, McDonald CI, McAuley DF, Fraser JF (2016) The inflammatory response to extracorporeal membrane oxygenation (ECMO): a review of the pathophysiology. Crit Care 20:387

33. Al-Fares A, Pettenuzzo T, Del Sorbo L (2019) Extracorporeal life support and systemic inflammation. Intensive Care Med Exp 7:46

34. Slutsky AS, Ranieri VM (2013) Ventilator-induced lung injury. N Engl J Med 369:2126-2136

35. Rozencwajg S, Guihot A, Franchineau G, Lescroat M, Brechot N, Hekimian G, Lebreton G, Autran B, Luyt CE, Combes A, Schmidt M (2019) UltraProtective ventilation reduces biotrauma in patients on venovenous extracorporeal membrane oxygenation for severe acute respiratory distress syndrome. Crit Care Med 47:1505-1512

36. Burrell AJC, Lubnow M, Enger TB, Nanjayya VB, Philipp A, Malfertheiner MV, Lunz D, Bein T, Pellegrino VA, Muller T (2017) The impact of venovenous extracorporeal membrane oxygenation on cytokine levels in patients with severe acute respiratory distress syndrome: a prospective, observational study. Crit Care Resusc 19:37-44

37. Burns S, Constantin N, Robles P, Investigators RP (2018) Understanding the long-term sequelae of ECMO survivors. Intensive Care Med 44:1144-1147

38. Vogel AM, Lew DF, Kao LS, Lally KP (2011) Defining risk for infectious complications on extracorporeal life support. J Pediatr Surg 46:2260-2264

39. O'Neill JM, Schutze GE, Heulitt MJ, Simpson PM, Taylor BJ (2001) Nosocomial infections during extracorporeal membrane oxygenation. Intensive Care Med 27:1247-1253

40. Danial P, Hajage D, Nguyen LS, Mastroianni C, Demondion P, Schmidt M, Bougle A, Amour J, Leprince P, Combes A, Lebreton G (2018) Percutaneous versus surgical femoro-femoral veno-arterial ECMO: a propensity score matched study. Intensive Care Med 44:2153-2161

41. Burket JS, Bartlett RH, Vander Hyde K, Chenoweth CE (1999) Nosocomial infections in adult patients undergoing extracorporeal membrane oxygenation. Clin Infect Dis 28:828-833

42. Kim DW, Yeo HJ, Yoon SH, Lee SE, Lee SJ, Cho WH, Jeon DS, Kim YS, Son BS, do Kim H (2016) Impact of bloodstream infections on catheter colonization during extracorporeal membrane oxygenation. J Artif Org 19:128-133

43. Kuehn C, Orszag P, Burgwitz K, Marsch G, Stumpp N, Stiesch M, Haverich A (2013) Microbial adhesion on membrane oxygenators in patients requiring extracorporeal life support detected by a universal rDNA PCR test. ASAIO J 59:368-373

44. Muller T, Lubnow M, Philipp A, Schneider-Brachert W, Camboni D, Schmid C, Lehle K (2011) Risk of circuit infection in septic patients on extracorporeal membrane oxygenation: a preliminary study. Artif Organs 35:E84-E90

45. Kanda H, Kubo K, Hamasaki K, Kanda Y, Nakao A, Kitamura T, Fujita T, Yamamoto K, Mimura T (2001) Influence of various hemodialysis membranes on the plasma (1->3)-beta-D-glucan level. Kidney Int 60:319-323

46. Glater-Welt LB, Schneider JB, Zinger MM, Rosen L, Sweberg TM (2016) Nosocomial bloodstream infections in patients receiving extracorporeal life support: variability in prevention practices: a survey of the extracorporeal life support organization members. J Intensive Care Med 31:654-669

47. Tangden T, Ramos Martin V, Felton TW, Nielsen El, Marchand S, Bruggemann RJ, Bulitta JB, Bassetti M, Theuretzbacher U, Tsuji BT, Wareham DW, Friberg LE, De Waele JJ, Tam VH, Roberts JA, Infection Section for the European Society of Intensive Care Medicine tP, Pharmacodynamics Study Group of the European Society of Clinical M, Infectious Diseases tISOA-IP, the Critically III Patients Study Group of European Society of Clinical M, Infectious D (2017) The role of infection models and PK/PD modelling for optimising care of critically ill patients with severe infections. Intensive Care Med 43:1021-1032

48. Kao LS, Fleming GM, Escamilla RJ, Lew DF, Lally KP (2011) Antimicrobial prophylaxis and infection surveillance in extracorporeal membrane oxygenation patients: a multi-institutional survey of practice patterns. ASAIO J 57:231-238

49. Extracorporeal Life Support Organization (ELSO) (2012) ELSO Task Force on Infectious Disease on ECMO: diagnosis, treatment and prevention. Ann Arbor, Michigan. https://www.elso.org/AboutUs/TaskForces/Infec tiousDiseaseTaskForce.aspx. Accessed 21 Aug 2019

50. Eggimann P, Pagani JL, Dupuis-Lozeron E, Ms BE, Thevenin MJ, Joseph C, Revelly JP, Que YA (2019) Sustained reduction of catheter-associated bloodstream infections with enhancement of catheter bundle by chlorhexidine dressings over 11 years. Intensive Care Med 45:823-833

51. Abrams D, Garan AR, Brodie D (2019) Awake and fully mobile patients on cardiac extracorporeal life support. Ann Cardiothorac Surg 8:44-53

52. Burrell AJC, Bennett V, Serra AL, Pellegrino VA, Romero L, Fan E, Brodie D, Cooper DJ, Kaye DM, Fraser JF, Hodgson CL, International EN (2019) Venoarterial extracorporeal membrane oxygenation: a systematic review of selection criteria, outcome measures and definitions of complications. J Crit Care 53:32-37

53. Hodgson CL, Burrell AJC, Engeler DM, Pellegrino VA, Brodie D, Fan E, International EN (2019) Core outcome measures for research in critically ill patients receiving extracorporeal membrane oxygenation for acute respiratory or cardiac failure: an international, multidisciplinary, modified Delphi consensus study. Crit Care Med 47:1557-1563

54. Lorusso R, Alexander P, Rycus P, Barbaro R (2019) The extracorporeal life support organization registry: update and perspectives. Ann Cardiothorac Surg 8:93-98

55. Corley A, Lye I, Lavana JD, Ahuja A, Anstey CM, Jarrett P, Haisz E, Parke R, Pellegrino V, Buscher H, Fraser JF, Investigators EPS (2019) Nosocomial infection prevalence in patients undergoing extracorporeal membrane oxygenation (ECMO): protocol for a point prevalence study across Australia and New Zealand. BMJ Open 9:e029293

56. Shekar K, Roberts JA, Welch S, Buscher H, Rudham S, Burrows F, Ghassabian S, Wallis SC, Levkovich B, Pellegrino V, McGuinness S, Parke R, Gilder E, Barnett AG, Walsham J, Mullany DV, Fung YL, Smith MT, Fraser JF (2012) ASAP ECMO: antibiotic, sedative and analgesic pharmacokinetics during extracorporeal membrane oxygenation: a multi-centre study to optimise drug therapy during ECMO. BMC Anesthesiol 12:29 